\title{
Design an Early Detection and Classification for Diabetic Retinopathy by Deep Feature Extraction based Convolution Neural Network
}

\author{
Akey Sungheetha, \\ Data Science SIG member, \\ Computer Science and Engineering, \\ School of Electrical Engineering and Computing, \\ Adama Science and Technology University, \\ Adama, Nazret, Ethiopia. \\ Rajesh Sharma R, \\ Image Processing SIG member, \\ Computer Science and Engineering, \\ School of Electrical Engineering and Computing, \\ Adama Science and Technology University, \\ Adama, Nazret, Ethiopia.
}

\begin{abstract}
Early identification of diabetics using retinopathy images is still a difficult challenge. Many illness diagnosis techniques are accomplished by using the blood vessels present in fundus images. Many conventional methods fail to detect Hard Executes (HE) present in retinopathy images, which are used to determine the severity of diabetes disease. To overcome this challenge, the proposed research work extracts the features by incorporating deep networks through convolution neural networks $(\mathrm{CNN})$. The micro aneurysm may be seen in the early stages of the transformation from normal to sick condition on the images for mild DR. The level of severity of the diabetes condition may be classified by using the confusion matrix detection results. The early detection of the diabetic condition has been achieved through the HE spotted in the blood vessel of an eye by using the proposed CNN framework. The proposed framework is also used to detect a person's diabetic condition. This article consisting of proof for the accuracy of the proposed framework is higher than other traditional detection algorithms.
\end{abstract}


Journal of Trends in Computer Science and Smart technology (TCSST) (2021)

Vol.03/ No. 02

Pages: 81-94

https://www.irojournals.com/tcsst/

DOI: https://doi.org/10.36548/jtcsst.2021.2.002

\section{Keywords: Diabetic retinopathy, Convolutional Neural Network}

\section{INTRODUCTION}

In diabetic retinopathy [DR], most of the early warning signs and symptoms do not show up. Many medical tests are available to diagnose the disease. One such test is fundus image analysis, in which the experts may assess blood vessels, nerve tissue problems and other necessary items [1]. Diabetes mellitus is a metabolic disease of the body, which is also known as diabetes mellitus, a condition that occurs when the blood sugar levels are elevated. It is chronic and can damage numerous body organs, including the eyes. Diabetic retinopathy is an eye disease caused by excessive blood sugar levels.

It is a disease connected with diabetes and has caused irreversible vision impairment in the human eye [2]. The prediction is most useful in the early stages of diabetic retinopathy. The image of diabetes in the retinal fundus is commonly found $[3,4]$. The complete blindness can happen due to Diabetic retinopathy (DR). DR is a progressive procedure and thus the medical experts suggest that people with diabetes should be examined at least twice a year for the indications of the illness. Detection primarily depends on the fact that, in current clinical diagnosis, the visual scientist will analyze the colour picture of the fundus. This detection is time-consuming and tedious, which can also lead to a major mistake $[5,6]$.

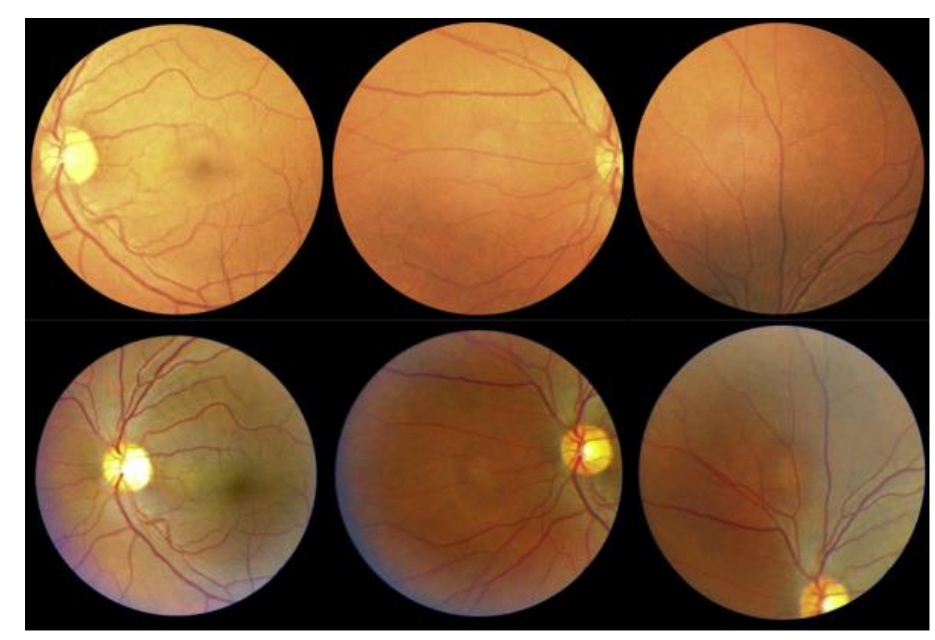

Figure 1 Samples of dataset retinal images 
Journal of Trends in Computer Science and Smart technology (TCSST) (2021)

Vol.03/ No. 02

Pages: 81-94

https://www.irojournals.com/tcsst/

DOI: https://doi.org/10.36548/jtcsst.2021.2.002

Figure 1 displays a sample set of data-set will different retinal images. Moreover, in certain areas, many DR patients do not diagnose and treat them early and lose their best therapeutically possibilities and have irreversible vision loss [7,8], due to the absence of healthcare resources. The process can be handled and deferred effectively, especially for the early-stage disorders, if the DR is immediately recognized and treated. The effect of manual interpretation is determined by the experience of the clinician. Misdiagnosis is common when people have not consulted their doctors. [9]. Figure 2 shows the normal and abnormal retina images in a single picture.

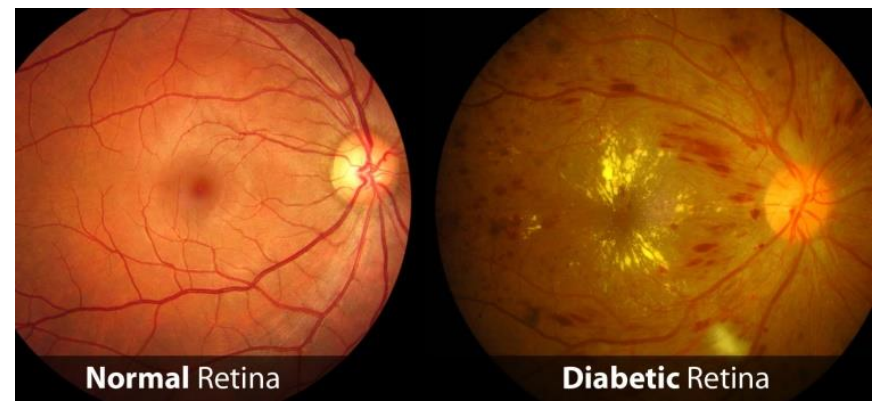

Figure 2 dataset containing both normal and abnormal retina

Current ICT advancements will have a significant impact on health improvement. Images may be identified and interpreted and further the data representation that supports the measures $[10,11]$ in integrated computer vision mainly in the context of artificial intelligence [12]. In the field of healthcare, promising outcomes are shown. The profound learning discipline comprises of a layered and data analysis-friendly design. With the increasing complexity of medical imaging, computers can categorize the medical images by employing a wide range of processes such as analysis, recognition, identification, and classification by using a deep learning architecture $[13,14]$. It requires a considerable expertise in less privileged sections.

These difficulties can be overcome through the automated diabetic retinopathy grading $[15,16]$. A number of techniques have been suggested in this sector, where profound learning seems to yield promising results. Conventional processes such as fluorescence approaches, morphological operations, random forest classification, etc. have been proven to exaggerate deep learning algorithms. However, in order to achieve better results, a large computer power and a dataset repository are required $[17,18]$. 
Journal of Trends in Computer Science and Smart technology (TCSST) (2021)

Vol.03/ No. 02

Pages: 81-94

https://www.irojournals.com/tcsst/

DOI: https://doi.org/10.36548/jtcsst.2021.2.002

\section{ORGANIZATION OF THE RESEARCH}

Structure of this research article is pre-arranged as follows; section 3 delivers recent related works of early detection techniques of diabetic retinopathy analysis. Section 4 provides the idea about methodology for detecting diabetic condition in early stage. Section 5 discusses about obtained results. Section 6 consists of conclusion and future task of our research works.

\section{PRELIMINARIES}

For diabetic retinopathy analysis, Yang et al. suggested a two-stage deep CNN approach for accurate detection. The algorithm shows the kind of lesions in fundus pictures, while the severity grade of each image is determined. The performance of their suggested method was further enhanced with the use of an uneven weighting map. In EyePACS data, lesion patches of $12 \mathrm{~K}$ were marked and the grades of pictures of $23,5 \mathrm{~K}$ were recalculated. The exactness of their method presented was 95.95\% [19].

In 2020, Gayatri et al proposed work on the extraction of Haralick and Anisotropic Complex Wavelet Tree Process (ADTCWT) multiple classifiers are evaluated such as Support Vector Machine (SVM), Random Forest, Random Tree, and J48. And Random Forest has been proven to exceed all other classifications with total accuracy of 99.7 percent. In 2020 Gayatri S. and others recommended a lightweight $\mathrm{CNN}$ for the DR categorization from fundus pictures. And the findings analyzed to demonstrate that the suggested extraction technology is superior in conjunction [20, 21]. RaghavVenkatesan et al developed an improved autocorrelogram (AutoCC) color function with low dimensionality in recently [22]. Madhusudan $\mathrm{R}$ et al introduced a model by integrating artificial intelligence to identify multi-class retinal disorders. Mindful data is used for training CNN in the suggested model [23].

A Diabetic Retinopathy CAD framework based on fondus photographs employing deep CNN imaging was suggested by shih-jen chen et al in 2020 . They proposed a common method to handle the database imbalance training, in which the techniques are employed for weighing the samples at high cost in uncommon classes [24].

For second and fifth category based diabetic retinopathy issues, Gosh et al. built a CNN model based on a collection of 30,000 image data with an accuracy of $95 \%$ and a precision of 
Journal of Trends in Computer Science and Smart technology (TCSST) (2021)

Vol.03/ No. 02

Pages: 81-94

https://www.irojournals.com/tcsst/

DOI: https://doi.org/10.36548/jtcsst.2021.2.002

$85 \%$ [25]. They used denoising as a pre-processing method. In 2017, Ardiyanto et al. suggested loading a compact Deep-DR-net algorithm in tiny embedded boards. Their profound learning system was designed to allow future embedded low-cost devices to detect high-performance diseases [26].

Many research works have examined and analyzed new state-of-the-art DR colour fondus image identification and classification algorithms by utilizing profound learning approaches. A variety of machine learning approaches, including the Random Forest, the SVM, etc., were employed to improve the literature's performance of DR detection. However, many significant characteristics that might help to identify a DR picture remain to be explored [27].

\section{METHODOLOGIES}

The deep learning CNN method is one of the powerful tools in detection activities. Recently, this approach is performing efficiently in image data classification, particularly retinal fundus image analysis. This research article proposes the variation of CNN model in the retinal fundus images in order to detect diabetic conditions at an early stage.

\section{Proposed Framework}

In our proposed algorithm, the DR is categorized into several stages as follows;

1. No DR

2. Mild DR

3. Moderate DR

4. Severe DR

5. Proliferative DR

This class separation is done through the proposed algorithm at first and then it considers the first stage of DR by mild class. Figure 3 shows the ultimate objective of proposed work.

\begin{tabular}{|c|c|c|}
\hline $\begin{array}{c}\text { DR level identification task of DR } \\
\text { severity classification in Fundus } \\
\text { Images }\end{array}$ & $\begin{array}{c}\text { DR Severity Classification based } \\
\text { on Confusion matrix values }\end{array}$ \\
\cline { 3 - 3 }
\end{tabular}

Figure 3 Proposed work 
Journal of Trends in Computer Science and Smart technology (TCSST) (2021)

Vol.03/ No. 02

Pages: 81-94

https://www.irojournals.com/tcsst/

DOI: https://doi.org/10.36548/jtcsst.2021.2.002

\section{$\underline{\text { Step 1: }}$}

Pre-processing of each image in datasets of fundus images

\section{Step 2:}

Features extraction

\section{Step 3:}

Feature fusion through dense deep

Feature fusion applying through deep features extracted from each image.

The 2 max pooling and average cross pooling can be performed for dense deep valuation. The feature vector is identified and classified through training.

\section{Step 4:}

Model training by extracted deep feature

\section{Step 5:}

Model evaluation through the validation process

At the early stage, the microaneurysms form on the blood vessels in the eyes from the transformation of normal to abnormal. At the moderate stage, the blood vessel gets swelling in DR. Severity level can be observed abnormal growth of blood vessels. This condition has contained some blockages in the huge amount of blood vessels. Finally, the DR at the last stage is failed the complete vision. This retinal detachment is combined with a huge amount of retinal break leads the vision loss [28]. This early-stage identification is focused to classify the spot of "HE" and concluding the diabetic condition of the patient.

Case 1 The DR is identifying successfully by the proposed framework with the highest accuracy. Input image of the patients' needs to check DR conditions that can be done through binary classification approach. The loss is measured through this binary cross-entropy of the object function. Then this class can be labelled as positive or negative based on the conditions.

Case 2 DR severity level prediction can be done through our proposed framework rather than the traditional method

During the process of DR-affected images, the identification understands the level of severity for a better treatment process to optimize the objective function. Figure 4 shows the work flow organization by our proposed framework. 
Journal of Trends in Computer Science and Smart technology (TCSST) (2021)

Vol.03/ No. 02

Pages: 81-94

https://www.irojournals.com/tcsst/

DOI: https://doi.org/10.36548/jtcsst.2021.2.002

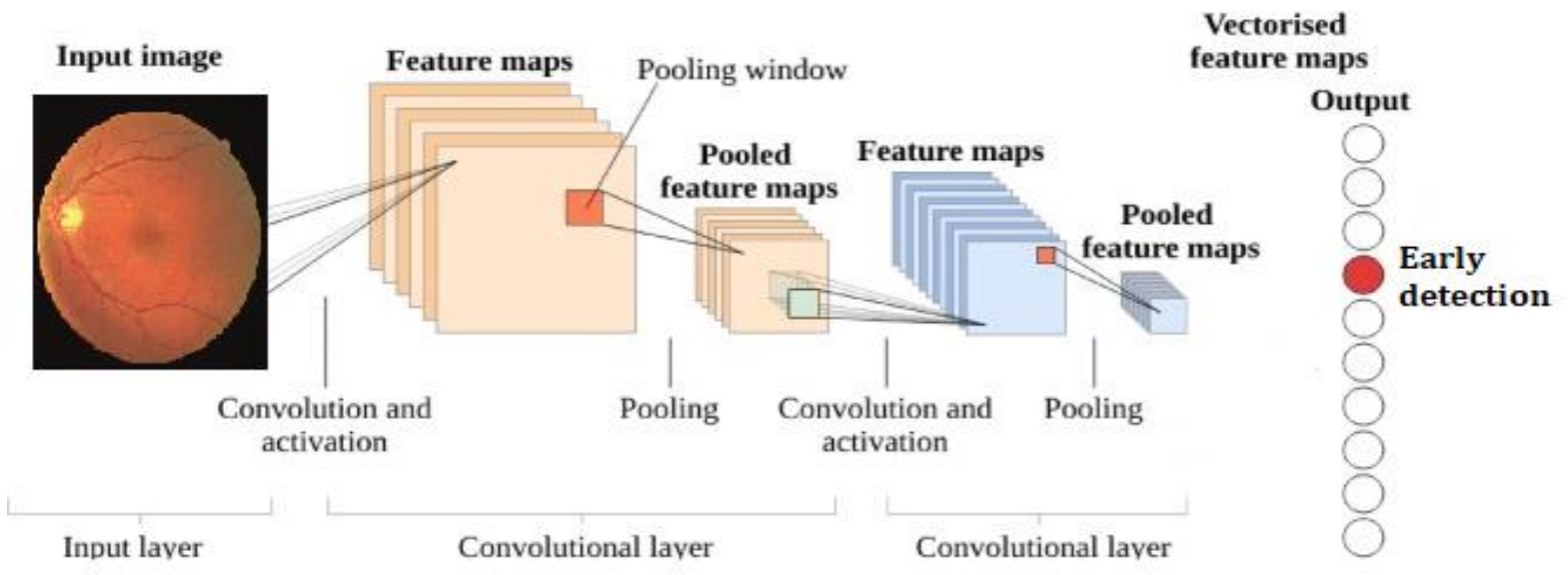

Figure 4 Work Flow of Proposed Framework

\section{RESULTS DISCUSSION}

This examination has been conducted by retinal images through modal features that are obtained by our proposed dense deep features of CNN. This evaluation can be compared with existing classification methods. Figure 5 shows identification levels of diabetic retinopathy through confusion matrix.

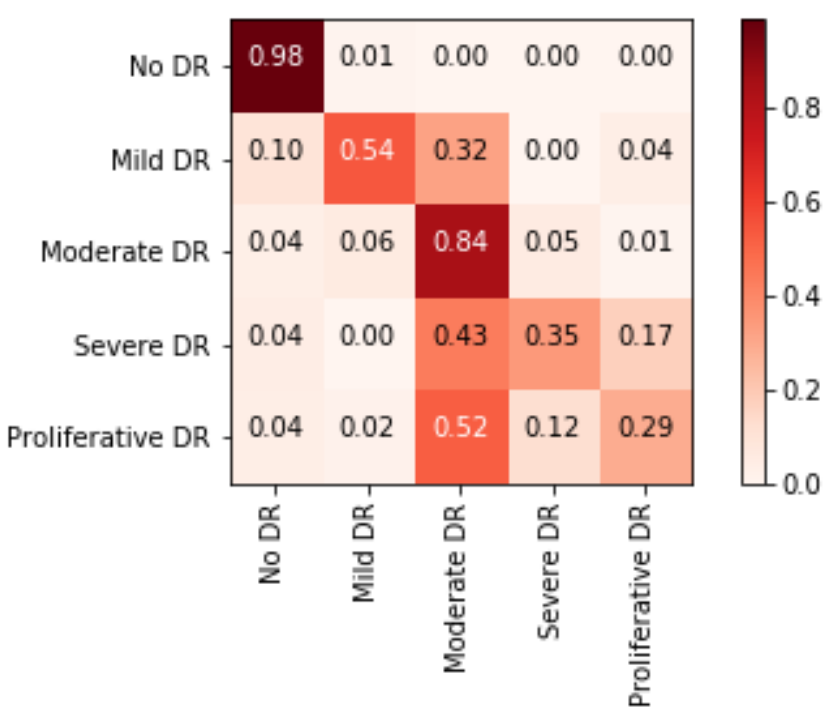

Figure 5 The Proposed Algorithm Identification for Different Levels 
Journal of Trends in Computer Science and Smart technology (TCSST) (2021)

Vol.03/ No. 02

Pages: 81-94

https://www.irojournals.com/tcsst/

DOI: https://doi.org/10.36548/jtcsst.2021.2.002

The performance measures used here are accuracy precision, recall, and F1 score. Newly, this research work has added the another performance metric named accuracy, the acuracy score of the proposed model is defined as,

$$
\text { class score }=\frac{\text { Observed accuracy }- \text { expected accuracy }}{1-\text { expected accuracy }}
$$

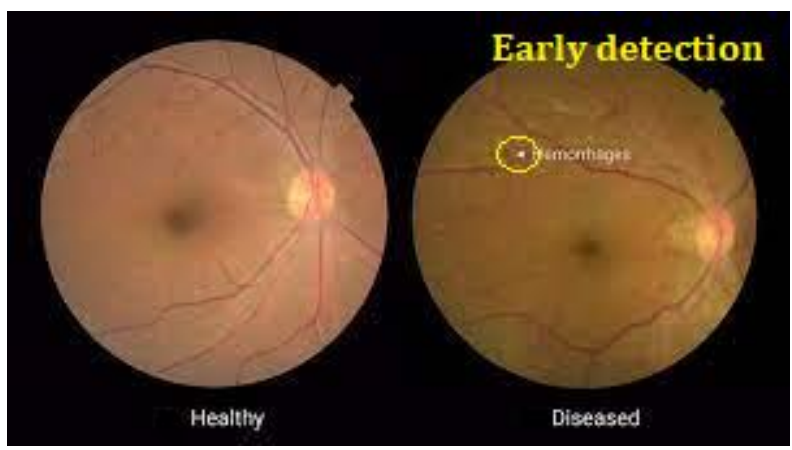

Figure 6 Early detection by probability based CNN approach

Figure 6 shows the obtained retinal images with early identification of diseased condition.

The yellow circle shows the HE and it is spotting by our proposed algorithm efficiently through several images where traditional method fails. It is possible to identify an early detection, when $\mathrm{CNN}$ is responsible of performing densed deep feature extraction. In general, it is defined as the number of samples that are properly categorized. . The expected accuracy we mentioned was $100 \%$ accuracy rate. The predicted value is satisfied with very little correction label. The other performance metrics are defined as follows;

$$
\begin{gathered}
\text { Accuracy }=\frac{T P+T N}{T P+T N+F P+F N} \\
\text { Precision }=\frac{T P}{T P+F P} \\
\text { Specificity }=\frac{T N}{T N+F P} \\
\text { Recall }=\frac{T P}{T P+F N}
\end{gathered}
$$


Journal of Trends in Computer Science and Smart technology (TCSST) (2021)

Vol.03/ No. 02

Pages: 81-94

https://www.irojournals.com/tcsst/

DOI: https://doi.org/10.36548/jtcsst.2021.2.002

Table 1 shows performance metric computation of our proposed framework.

Table 1 computed class performances

\begin{tabular}{|c|c|c|c|c|c|c|}
\hline S.No & Methods & Recall & Precision & Specificity & Accuracy & $\begin{array}{c}\text { Early } \\
\text { detection } \\
\mathbf{( \% )}\end{array}$ \\
\hline 1 & Image processing & $86 \%$ & $79 \%$ & $81.5 \%$ & $80 \%$ & $50 \%$ \\
\hline 2 & $\begin{array}{c}\text { Single classifier } \\
\text { (Naïve Bayes) }\end{array}$ & $80 \%$ & $77 \%$ & $80 \%$ & $75 \%$ & $60 \%$ \\
\hline 3 & $\begin{array}{c}\text { Single classifier } \\
\text { (SVM) }\end{array}$ & $90 \%$ & $88 \%$ & $89 \%$ & $94 \%$ & $65 \%$ \\
\hline 4 & $\begin{array}{c}\text { Proposed deep } \\
\text { features CNN }\end{array}$ & $92 \%$ & $89.5 \%$ & $92 \%$ & $97 \%$ & $96 \%$ \\
\hline
\end{tabular}

Figure 7 shows the graph plotting the obtained performance measure parameters for our proposed framework.

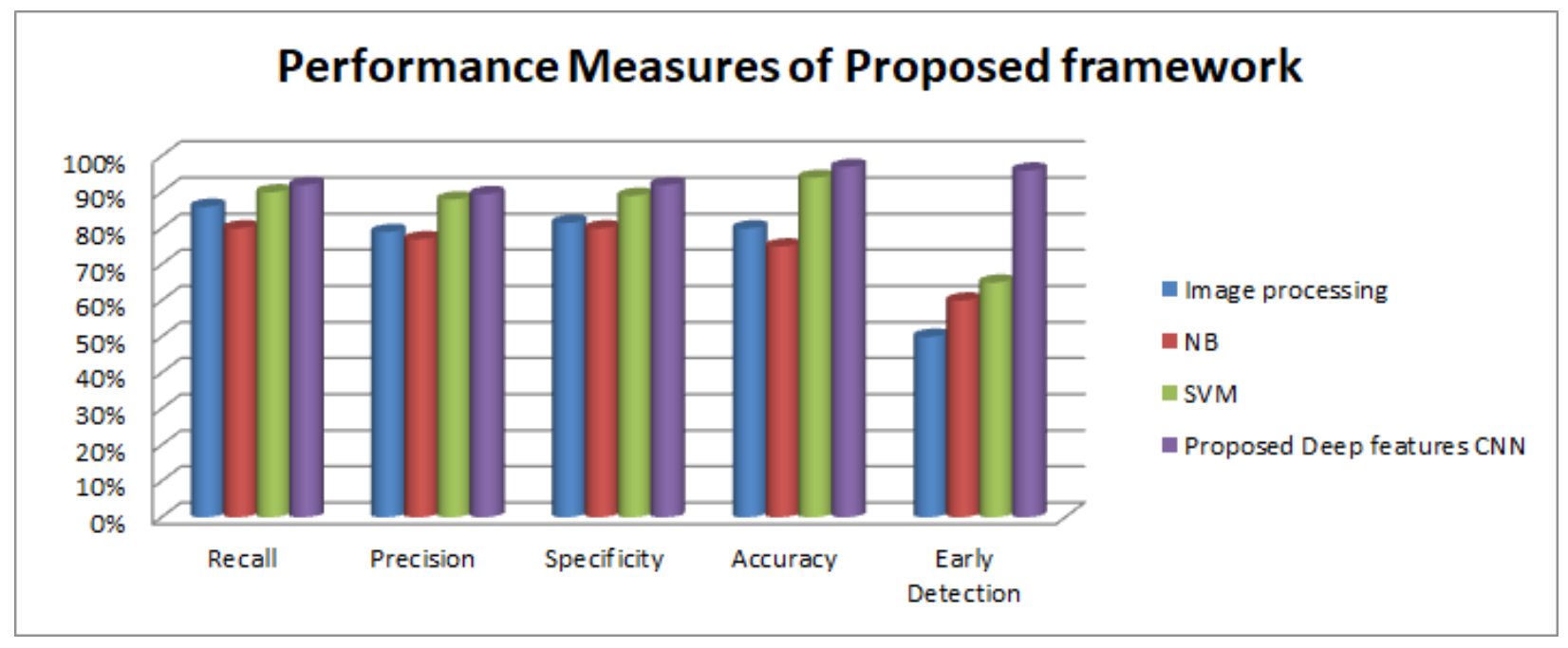

Figure 7 Overall performance measures of proposed framework

The graph shows the proposed framework class performance computation that shows the superiority than other methods. An early detection can be performed in well manner by using our proposed frame work. All other fundamental performance parameters show the the performance of the proposed method work is good and it is greater than the other existing methods. 
Journal of Trends in Computer Science and Smart technology (TCSST) (2021)

Vol.03/ No. 02

Pages: 81-94

https://www.irojournals.com/tcsst/

DOI: https://doi.org/10.36548/jtcsst.2021.2.002

\section{CONCLUSION}

Thus, the proposed framework has detected the spot of HE in the blood vessels of the retinal images by performing a dense deep feature extraction. The early detection can be performed in a very successful and efficient manner by using the $\mathrm{CCN}$ based deep learning classification. The performance metrics for the proposed algorithm has been tabulated and further it is compared with the traditional approach for the detection of diabetic retinopathy from the input image. The primary objective of this study is to employ transfer education in order to improve previously learned task network parameters for performing enhanced image categorization. In the future, the suggested architecture will be extended to include images from the unfiltered world in real-time. For real situations in clinical applications, further testing is essential and the system should be robust. Such approaches might enable healthcare practitioners to consult more patients to promptly diagnose them. This will mean that, the incorporation of large archives into deep learning systems will be highly essential in the near future [29, 30].

\section{REFERENCES}

[1] Sekar, K. R., S. Yogapriya, N. Senthil Anand, and V. Venkataraman. "Ranking Diabetic Mellitus Using Improved PROMETHEE Hesitant Fuzzy for Healthcare Systems." In Intelligent Data Communication Technologies and Internet of Things: Proceedings of ICICI 2020, pp. 709-724. Springer Singapore, 2021.

[2] Hamdan, Yasir Babiker. "Faultless Decision Making for False Information in Online: A Systematic Approach." Journal of Soft Computing Paradigm (JSCP) 2, no. 04 (2020): 226-235.

[3] Yaganteeswarudu, Akkem, and Prabhakar Dasari. "Diabetes Analysis and Risk Calculation-Auto Rebuild Model by Using Flask API." In International Conference on Image Processing and Capsule Networks, pp. 299-308. Springer, Cham, 2020.

[4] Raumviboonsuk, P.; Krause, J.; Chotcomwongse, P.; Sayres, R.; Raman, R.;Widner, K.; Campana, B.J.L.; Phene, S.; Hemarat, K.; Tadarati, M.; et al. Deep learning versus human graders for classifying diabetic retinopathy severity in a nationwide screening program. NPJ Digit. Med. 2019, 2, 1-9. 
Journal of Trends in Computer Science and Smart technology (TCSST) (2021)

Vol.03/ No. 02

Pages: 81-94

https://www.irojournals.com/tcsst/

DOI: https://doi.org/10.36548/jtcsst.2021.2.002

[5] Kumar, Silpa Ajith, and J. Satheesh Kumar. "A review on recent developments for the retinal vessel segmentation methodologies and exudate detection in fundus images using deep learning algorithms." In International Conference On Computational Vision and Bio Inspired Computing, pp. 1363-1370. Springer, Cham, 2019.

[6] Haoxiang, Wang, and S. Smys. "Overview of Configuring Adaptive Activation Functions for Deep Neural Networks-A Comparative Study." Journal of Ubiquitous Computing and Communication Technologies (UCCT) 3, no. 01 (2021): 10-22.

[7] Abràmoff, M.D.; Lou, Y.; Erginay, A.; Clarida, W.; Amelon, R.; Folk, J.C.; Niemeijer, M. Improved automated detection of diabetic retinopathy on a publicly available dataset through integration of deep learning. Investig. Ophthalmol. Vis. Sci. 2016, 57, 52005206.

[8] Koresh, H. James Deva, and Shanty Chacko. "Hybrid speckle reduction filter for corneal OCT images." In International Conference on Image Processing and Capsule Networks, pp. 87-99. Springer, Cham, 2020.

[9] Adam, Edriss Eisa Babikir. "Survey on Medical Imaging of Electrical Impedance Tomography (EIT) by Variable Current Pattern Methods." Journal of ISMAC 3, no. 02 (2021): 82-95.

[10] Retinopathy, A.D. Diabetic Retinopathy Detection using Deep Convolutional Neural Networks. In Proceedings of the International Conference on Computing, Analytics and Security Trends, Pune, India, 19-21 December 2016.

[11] Kumar, R. Raja, R. Pandian, T. Prem Jacob, A. Pravin, and P. Indumathi. "Detection of Diabetic Retinopathy Using Deep Convolutional Neural Networks." In Computational Vision and Bio-Inspired Computing, pp. 415-430. Springer, Singapore, 2021.

[12] Shakya, Subarna, and S. Smys. "Reliable Automated Software Testing Through Hybrid Optimization Algorithm." Journal of Ubiquitous Computing and Communication Technologies (UCCT) 2, no. 03 (2020): 126-135.

[13] Shankar, K.; Zhang, Y.; Liu, Y.; Wu, L.; Chen, C.H. Hyperparameter tuning deep learning for diabetic retinopathy fundus image classification.IEEE Access 2020, 8, 118164-118173. 
Journal of Trends in Computer Science and Smart technology (TCSST) (2021)

Vol.03/ No. 02

Pages: 81-94

https://www.irojournals.com/tcsst/

DOI: https://doi.org/10.36548/jtcsst.2021.2.002

[14] Adam, Edriss Eisa Babikir. "Evaluation of Fingerprint Liveness Detection by Machine Learning Approach-A Systematic View." Journal of ISMAC 3, no. 01 (2021): 16-30.

[15] Shu, D.; Ting, W.; Cheung, C.Y.L.; Lim, G.; Siew, G.; Tan, W.; Quang, N.D.; Gan, A.; Hamzah, H.; Garcia-franco, R.; et al. Development and Validation of a Deep Learning System for Diabetic Retinopathy and Related Eye Diseases Using Retinal Images From Multiethnic Populations With Diabetes. JAMA 2017, 318, 2211-2223.

[16] Karuppusamy, P. "Building Detection using Two-Layered Novel Convolutional Neural Networks." Journal of Soft Computing Paradigm (JSCP) 3, no. 01 (2021): 29-37.

[17] Suriyal, S.; Druzgalski, C.; Gautam, K. Mobile assisted diabetic retinopathy detection using deep neural network. In Proceedings of the 2018 Global Medical Engineering Physics Exchanges/Pan American Health Care Exchanges (GMEPE/PAHCE), Porto, Portugal, 19-24 March 2018; pp. 1-4.

[18] Hariharakrishnan, Jayaram, and N. Bhalaji. "Adaptability Analysis of 6LoWPAN and RPL for Healthcare applications of Internet-of-Things." Journal of ISMAC 3, no. 02 (2021): 69-81.

[19] Yang, Y.; Li, T.; Li, W.; Wu, H.; Fan, W.; Zhang, W. Lesion Detection and Grading of Diabetic Retinopathy via Two-Stages Deep Convolutional Neural Networks. pp. 1-8. Available online: http://xxx.lanl.gov/abs/arXiv:1705.00771v1

[20] S. Gayathri, A. K. Krishna, V. P. Gopi, and P. Palanisamy. "Automated binary and multiclass classification of diabetic retinopathy using haralick and multiresolution features" IEEE Access, 8:57497-57504, 2020.

[21] Gayathri S., Varun P. Gopi, and P. Palanisamy. A lightweight cnnfor diabetic retinopathy classification from fundus images. Biomedical Signal Processing and Control, 62:102115, 2020.

[22] R. Venkatesan, P. Chandakkar, B. Li, and H. K. Li. Classification ofdiabetic retinopathy images using multi-class multiple-instance learningbased on color correlogram features. In 2012 Annual InternationalConference of the IEEE Engineering in Medicine and Biology Society,pages 1462-1465, 2012. 
Journal of Trends in Computer Science and Smart technology (TCSST) (2021)

Vol.03/ No. 02

Pages: 81-94

https://www.irojournals.com/tcsst/

DOI: https://doi.org/10.36548/jtcsst.2021.2.002

[23] Madhusudan R. J Sundaramoorthy S. K Namboori P. K. K.Karthikeyan S., Kumar P. S. Detection of multi-class retinal diseasesusing artificial intelligence: An expeditious learning using deep cnn withminimal data. Biomed Pharmacol J, 2019.

[24] Shih-Jen Chen 2 Yung-Hui Li, Nai-Ning Yeh and Yu-Chien Chung3.Computerassisted diagnosis for diabetic retinopathy based on fundus images using deep convolutional neural network. Mobile InformationSystems, 2020.

[25] Ghosh, R. Automatic Detection and Classification of Diabetic Retinopathy stages using CNN. In Proceedings of the $20174^{\text {th }}$ International Conference on Signal Processing and Integrated Networks (SPIN), Noida, Delhi-NCR, India, 2-3 February 2017.

[26] Ardiyanto, I.; Nugroho, H.A.; Lestari, R.; Buana, B. Deep Learning-based Diabetic Retinopathy Assessment on Embedded System. In Proceedings of the 2017 39th Annual International Conference of the IEEE Engineering in Medicine and Biology Society (EMBC), Jeju Island, Korea, 11-15 July 2017; pp. 1760-1763.

[27] Manoharan, J. Samuel. "A Novel User Layer Cloud Security Model based on Chaotic Arnold Transformation using Fingerprint Biometric Traits." Journal of Innovative Image Processing (JIIP) 3, no. 01 (2021): 36-51.

[28] Kajan, S.; Goga, J.; Lacko, K.; Pavlovi`cová, J. Detection of Diabetic Retinopathy Using Pretrained Deep Neural Networks. In Proceedings of the 2020 Cybernetics \& Informatics (K\&I), Velké Karlovice, Czech Republic, 29 January-1 February 2020; pp. $1-5$.

[29] Vijayakumar, T., Mr R. Vinothkanna, and M. Duraipandian. "Fusion based Feature Extraction Analysis of ECG Signal Interpretation-A Systematic Approach." Journal of Artificial Intelligence 3, no. 01 (2021): 1-16

[30] Karunakaran, P., and Yasir Babiker Hamdan. "Early Prediction of Autism Spectrum Disorder by Computational Approaches to fMRI Analysis with Early Learning Technique." Journal of Artificial Intelligence 2, no. 04 (2020): 207-216. 


\section{Authors Biography}

Akey Sungheetha is a Data Science SIG member, in the department of Computer Science and Engineering, in School of Electrical Engineering and Computing, in Adama Science and Technology University, Adama, Nazret, Ethiopia. Akey Sungheetha has 16 years of academic experience. Her area of research is networking, and she has published in more than 50 international and national journals. She is a life member of International Association of Engineers and Indian Society of Technical Education, and member of IIE, IAENG, IARCP, ISQEM and AIIM.

Rajesh Sharma $\mathrm{R}$ has more than 8 years of academic experience. His areas of research are networking, probabilistic computing, fuzzy, bio- inspired computing, data visualization, fault diagnosis, robotics, internet of things, neuro computing, information retrieval human-machine interface and network security. He has published in more than 20 international and national journals. He is a life member of International Association of Engineers and Indian Society of Technical Education, and member of IAENG, IACSIT and AACE. 